\title{
A ORGANIZAÇÃO DE CAMPO DE UMA PESQUISA NACIONAL DE AVALIAÇÃO: RELATO DE EXPERIÊNCIA
}

\author{
Isabela Cristina Nogueira \\ Graduanda em Enfermagem pela Universidade Estadual de Campinas \\ n.isabela07@gmail.com
}

INTRODUÇÃO: O Programa Nacional de Melhoria do Acesso e da Qualidade da Atenção Básica (PMAQ-AB) tem como objetivo incentivar gestores e equipes a melhorar a qualidade dos serviços de saúde oferecidos aos cidadãos. A avaliação do PMAQ é realizada pelo Ministério da Saúde em parceria com as Instituições de Ensino e Pesquisa. OBJETIVO: Relatar a experiência da coordenação de campo de pesquisa do 3ํㅜ Ciclo do PMAQ-AB em municípios das regionais de Campinas e de São João da Boa Vista, estado de São Paulo, realizada por equipe composta por coordenadora docente da Faculdade de Enfermagem (Fenf) da Unicamp, assistente de pesquisa doutora em enfermagem e estagiária do último ano da graduação da FEnf/Unicamp. MÉTODO: A organização de campo abrange a comunicação com a coordenação geral do PMAQ-AB/SP, articulação com gestores municipais e estaduais da atenção básica, coordenação de três equipes de entrevistadores, planejamento e administração da logística do roteiro de pesquisa e monitoramento pelo sistema nacional de gestão da pesquisa. RESULTADOS: Dentre os 53 municípios sob responsabilidade da coordenação de Campinas, que contam com 527 equipes de saúde da família (com ou sem saúde bucal), foram realizadas, até o momento, entrevistas em 367 equipes em um período de três meses. As entrevistas abordam estrutura da unidade, processo de trabalho, satisfação dos usuários e funcionamento das equipes dos Núcleos Ampliados de Saúde da Família. Foram realizados quatro encontros com os entrevistadores e contatos cotidianos com os supervisores. Conclusão: A experiência permitiu reflexões sobre a importância do espaço potente da universidade com avaliações em saúde de âmbito nacional, de forma a possibilitar a implementação de novas estratégias na gestão de serviços de saúde. Ademais, evidencia-se o papel da FEnf/Unicamp na condução de pesquisa que avalia a complexidade da Atenção Básica, regional contribuindo para processos de melhoria do acesso e da qualidade da atenção no SUS. 
Palavras-chave: Atenção básica. Gestão em saúde. Avaliação de serviços de saúde. 